Nanostructuring polymers by soft lithography templates realized via ion sputtering

This content has been downloaded from IOPscience. Please scroll down to see the full text. 2005 Nanotechnology 162714

(http://iopscience.iop.org/0957-4484/16/11/041)

View the table of contents for this issue, or go to the journal homepage for more

Download details:

IP Address: 155.69.4.4

This content was downloaded on 24/08/2015 at 23:00

Please note that terms and conditions apply. 


\title{
Nanostructuring polymers by soft lithography templates realized via ion sputtering
}

\author{
Elisa Mele ${ }^{1}$, Francesca Di Benedetto ${ }^{1}$, Roberto Cingolani ${ }^{1}$, \\ Dario Pisignano $^{1}$, Andrea Toma ${ }^{2}$, Francesco Buatier de Mongeot $^{2}$, \\ Renato Buzio $^{2}$, Corrado Boragno ${ }^{2}$, Giuseppe Firpo ${ }^{2}$, \\ Valentina Mussi ${ }^{2}$ and Ugo Valbusa ${ }^{2}$ \\ ${ }^{1}$ National Nanotechnology Laboratory of INFM-CNR and Agilent Technologies joint lab, \\ Università di Lecce, via Arnesano, I-73100, Lecce, Italy \\ 2 Dipartimento di Fisica, Università di Genova, via Dodecaneso 33, I-16146, Genova, Italy \\ E-mail: elisa.mele@unile.it
}

Received 20 May 2005, in final form 4 August 2005

Published 6 October 2005

Online at stacks.iop.org/Nano/16/2714

\begin{abstract}
We demonstrate that a combination of ion sputtering and soft lithography is an alternative and effective way of nanostructuring soft matter. We create self-organized nanoscale structures on a glass template by irradiating the surface with a defocused, low energy Ar ion beam. Capillary force lithography is then used to transfer the pattern, exploiting the glass transition of polymeric layers. In particular, we demonstrate the pattern transfer of a periodic $150 \mathrm{~nm}$ ripple structure onto an organic compound. This new, unconventional combination is then a low-cost strategy that opens the way to a variety of applications in the field of organic-based devices.
\end{abstract}

(Some figures in this article are in colour only in the electronic version)

\section{Introduction}

Over the past decade, a number of lithographic techniques, such as electron and ion beams, $x$-rays, scanning probes [1], soft and imprinting lithographies [2-4], self-assembling [5], atom lithography [6], and micro-plasma discharges [7], have been developed as alternatives to photolithography for fabricating high quality micro- and nanostructures. In particular, soft lithographies embody the patterning methods (microcontact printing [5], replica molding [8], microtransfer molding [9], micromolding in capillaries [10], and solventassisted micromolding [11]), relying on the use of elastomeric replicas to transfer the pattern onto a substrate. The stamp, made of poly(dimethylsiloxane) (PDMS) or other compounds able to provide conformal contact, is used to replicate the geometry of a starting master, pouring the liquid prepolymer of the elastomer against the master surface, and curing it in situ. The elastic nature of the stamps allows one to produce faithful copies of the complex features of the master. The resolution achievable with the soft lithographic methods is strongly dependent on the starting master, and thus it is limited by the availability of techniques to produce masters with nanometer resolution.

Recently, low-energy ion sputtering has been proposed as a simple and powerful method to produce nanostructures having feature sizes on the scale of some tens of nanometers at low cost, on large areas [12-16]. Depending on the experimental parameters, such as the surface temperature, sputtering time (ion dose) and ion incidence angle, it is possible to force the growth of a self-organized ripple structure. Besides its applicability to almost all materials, the microscopic mechanisms involved in the growth of the nanopatterns are different between metals or semiconductors and glasses. As a general rule, these structures result from the balance between the erosion induced by the ion collisions and the diffusion of surface atoms displaced after the ion impact. Bradley and Harper have proposed a linear equation describing such a surface height evolution, $h(r, t)$, with balance between erosion 
and diffusion terms [17]:

$$
\frac{\partial h}{\partial t}=v_{x} \frac{\partial^{2} h}{\partial x^{2}}+v_{y} \frac{\partial^{2} h}{\partial y^{2}}-B \nabla^{2} \nabla^{2} h,
$$

where $v_{x, y}$ are the prefactors related to the curvature dependent erosion rate, and $B$ accounts for the surface diffusion effects. However, in crystalline metals and semiconductors, a key role is played by the surface diffusion of the defects (adatoms, vacancies) created by the ions. The consequence is that the nanopattern geometry can be tailored by the surface symmetry and it can be strongly dependent on the temperature and on the ion flux. In glasses, viscosity diffusion and ion-induced diffusion represent the most important mechanisms for the recovery of the ion damage. This implies a longer time for the development of the nanopattern, whose direction depends on the ion beam projection on the surface $[18,19]$.

In this work, we demonstrate that the combination of ion sputtering and soft lithography can be exploited for nanostructuring soft matter. We realized self-organized nanoscale structures on a glass template by irradiating the surface with a defocused, low energy Ar ion beam. Capillary force lithography is then used to transfer patterns exploiting the glass transition of polymeric layers. In particular, we demonstrate the pattern transfer of a periodic $150 \mathrm{~nm}$ ripple structure onto organic compounds.

\section{Experimental details}

The basic scheme of the CFL is summarized in figure 1 . Glass masters were fabricated by ion sputtering. Glass was chosen because it is inert, rigid and can be heated up to high temperatures without any damage to the nanopattern. The sample is sonicated in acetone in order to eliminate organic contaminants and rinsed in pure water, then the substrate is inserted in a UHV chamber equipped with a defocused microwave ECR plasma source. The sample is ion bombarded at a temperature of $300 \mathrm{~K}$ with $\mathrm{Ar}$ ions having energy of $800 \mathrm{eV}$. The ion flux, measured by a Faraday cup, is about $400 \mu \mathrm{A} \mathrm{cm}^{-2}$. The angle of incidence is fixed at $35^{\circ}$ with respect to the substrate normal. To prevent the charging of the sample, a W filament (Th coated) is positioned at the end of the source, thus ensuring efficient neutralization of the substrate. In these conditions, a rippled nanopattern is produced, with crests perpendicular to the ion beam projection on the sample (for a detailed morphological characterization by means of a scaling law approach see [20]). A PDMS replica (Sylgard 184, Dow Corning, Midland, MI), fabricated by the nanostructured templates by a standard replica molding procedure [2], was then placed in conformal contact onto a polymeric film cast on a substrate. In our experiments, $0.8 \times 10^{-3} \mathrm{M}$ chloroform solution of poly(methylmethacrylate) (PMMA) (Sigma-Aldrich, MO, average molecular weight of $120 \mathrm{k}$ ) was spin-coated at $1500 \mathrm{rpm}$ onto a silicon substrate. When the polymer is heated at $170^{\circ} \mathrm{C}$, a temperature value higher than $T_{\mathrm{g}}\left(120^{\circ} \mathrm{C}\right)$, capillary effects force the polymer melt into the void space of the features formed between the replica and the polymeric film. After the subsequent cooling to ambient temperature, the replica is peeled off, and the pattern remains on the target.
I. Contact between the elastomeric template and the thermoplastic film

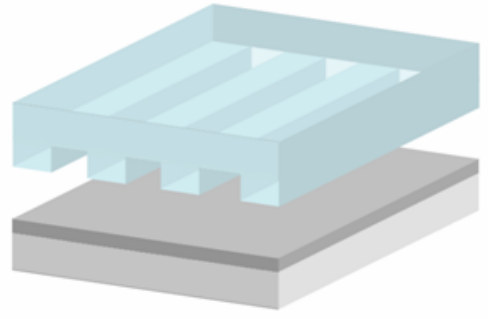

II. Filling of the void spaces of the features formed between the replica and the polymeric film by capillary force during heating above $T_{g}$.

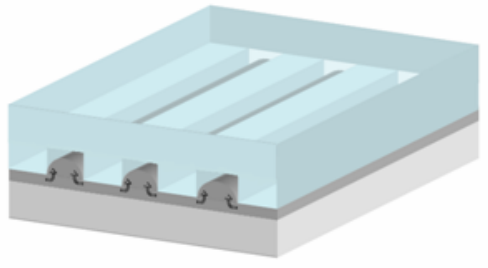

III. Template separation after cooling at room temperature

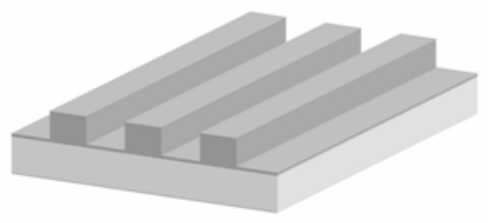

Figure 1. Schematic diagram of the capillary force lithography procedure by elastomeric template (features not in scale).

\section{Results and discussion}

Since the spatial periodicity as well as the depth of the troughs depends on the sputtering dose as shown in figure 2, the sputtering time was set at $100 \mathrm{~min}$ to obtain an average wavelength of about $200 \mathrm{~nm}$. Under these conditions the morphology of the glass surface is dominated by a quasiregular array of long channels, of depth of $(11.2 \pm 1.1) \mathrm{nm}$ (RMS). The crest to valley height modulation lies in the broad range $30-60 \mathrm{~nm}$, as can be appreciated in figure 3 . This is due to the stochastic fluctuations related to the self-assembly process. The ripple wavelength, $\lambda$, can be calculated from the two-dimensional map of the height-height correlation function $G(i, j)$ as described in [21]. $G(i, j)$ is defined as

$$
G(i, j)=\frac{1}{l^{2}} \sum_{m, n=1}^{l} h(i+m, j+n) h(i, j),
$$

where $h(i, j)$ denotes the surface height at position $(i, j)$. The AFM image of the nanopatterned glass is reported in figure 3.

Among the different soft lithography approaches, we chose the soft molding (SM [22]) or capillary force lithography (CFL [23]), which joins the advantages of nanoimprint lithography (NIL) and replica-assisted techniques. In the 


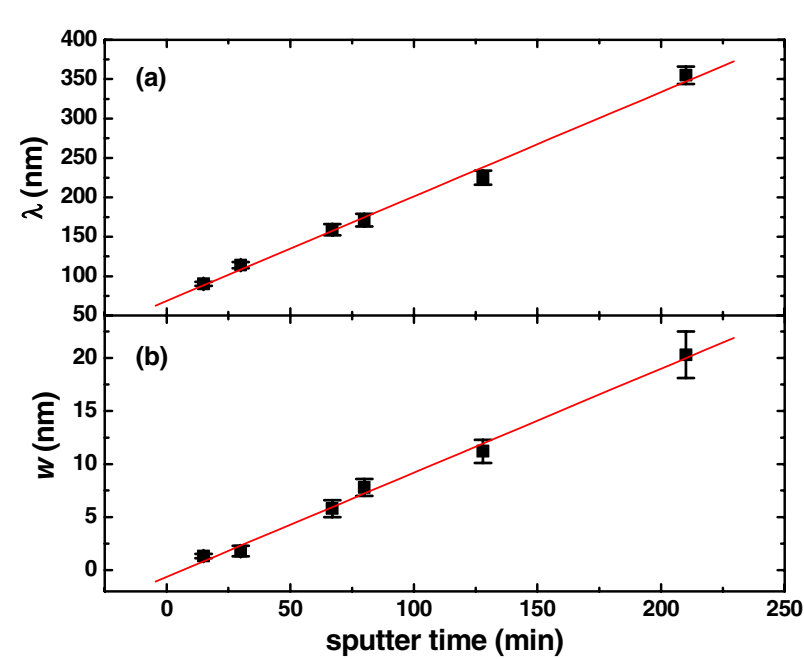

Figure 2. Ripple wavelength $\lambda$ (a) and RMS surface roughness $w$ (b) versus sputtering time $t$. The solid lines represent a linear fit according to the power law $\lambda=t^{\gamma}$ and $w=t^{\beta}$, with $\gamma=0.95 \pm 0.05$ and $\beta=1.02 \pm 0.20$ respectively.

CFL the key element of soft lithography, namely the use of an elastomeric replica, is combined with the working principle of NIL, i.e. the molding of a polymer melt. The conventional NIL consists of the compression molding of thermoplastic polymers. A hard mold with an engraved pattern is mechanically pressed into a thin film of a thermoplastic polymer, which becomes a viscous liquid, upon heating above the glass transition temperature $\left(T_{\mathrm{g}}\right)$. This determines the subsequent adaptation of the polymer to the mold pattern. To date, $10 \mathrm{~nm}$ diameter and $40 \mathrm{~nm}$ period holes have been fabricated in polymeric films by NIL [24]. Nevertheless, NIL presents a series of limitations, such as the difficult mold release, and the requirement of a very high pressure, typically on the order of $10^{6} \mathrm{~Pa}$ [24], to transfer the pattern. Moreover, the imprint quality is limited by the material transport, inherent to this technique, especially in the case of a negative stamp: the pattern transfer can be unsatisfactory and bubble-like defects can be observed, possibly due to a lack of conformal contact of the mold [25]. These limitations can be overcome by the use of a PDMS stamp. In fact, PDMS is chemically inert; consequently, it does not adhere irreversibly to the surface of the substrate, and its elastic characteristic allows the replica to spontaneously wet the surface, thus providing a conformal contact with the substrate, without any external force, over a large area.

The penetration of the polymer into the recessed features of the PDMS mold is driven by capillarity and favoured by a low polymer viscosity. In fact, the time $t$ taken for the filling process depends on the viscosity of the polymer melt, $\eta$, according to equation [26]:

$$
t=\frac{2 \eta z^{2}}{\gamma R \cos \Theta}
$$

where $z$ is the feature size, $\gamma$ the fluid-air surface tension, $R$ the hydraulic radius of the capillary, and $\Theta$ the contact angle between the liquid and the surface of the capillary. Moreover, above the glass transition temperature, the viscosity of many

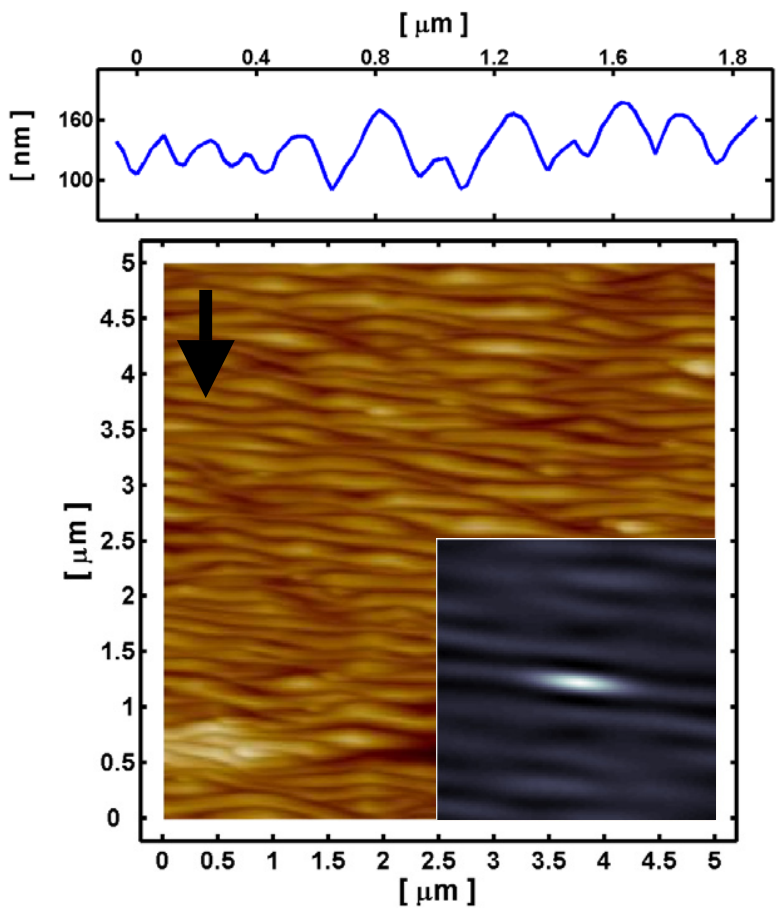

Figure 3. AFM image $\left(5 \times 5 \mu \mathrm{m}^{2}\right)$ of a sputter rippled glass and corresponding cross section along the ripple wavevector. The sputtering parameters were $E_{\text {ion }}=800 \mathrm{eV}, j_{\text {ion }}=400 \mu \mathrm{A} \mathrm{cm}^{-2}$, $\vartheta_{\text {ion }}=35^{\circ}, t=100 \mathrm{~min}$. The arrow indicates the projection of the ion beam. The inset reports the corresponding $G(i, j)$ (area $\left.2 \times 2 \mu \mathrm{m}^{2}\right)$.

polymers varies with the temperature [27]:

$$
\eta(T)=\eta_{0} \exp \left(\frac{D T_{0}}{T-T_{0}}\right),
$$

where $D$ is referred to as the strength parameter and $T_{0}$ is the temperature for which equation (4) shows a divergence in the polymer structural relaxation time and, consequently, in the viscosity. Therefore, a suitable temperature increment determines a dramatic decrease of the polymer viscosity, favoring the filling of the pattern. A few minutes are therefore enough for the pattern transfer. In figure 4, the AFM image of the polymeric pattern is displayed, showing a nanostructured polymer surface with a crest to valley height modulation in the range of 15-45 $\mathrm{nm}$, depending on the corresponding region of the master (i.e. with a loss of about $15 \mathrm{~nm}$ in the height profile). In particular, the achieved height of the imprinted features is strongly dependent on the imprinting time, and it can be increased by increasing the heating time during the CFL, which favors the complete capillarity filling of the void spaces of the PDMS template by the fluid polymer, thus accomplishing a faithful aspect ratio.

Finally, we have varied the morphology of the glass template in order to test the ability of the polymer to fill the profiles of the nanopattern under the action of capillary forces. Down-scaling the pattern to smaller pitches of the order $60 \mathrm{~nm}$ and to RMS depths of about $1.3 \mathrm{~nm}$ is easily achieved on glass substrates by reducing the irradiation dose to about $15 \mathrm{~min}$. Even smaller pitches of about $30 \mathrm{~nm}$ can be achieved by selecting different substrates such as LiF and even 
Nanostructuring polymers by soft lithography templates realized via ion sputtering
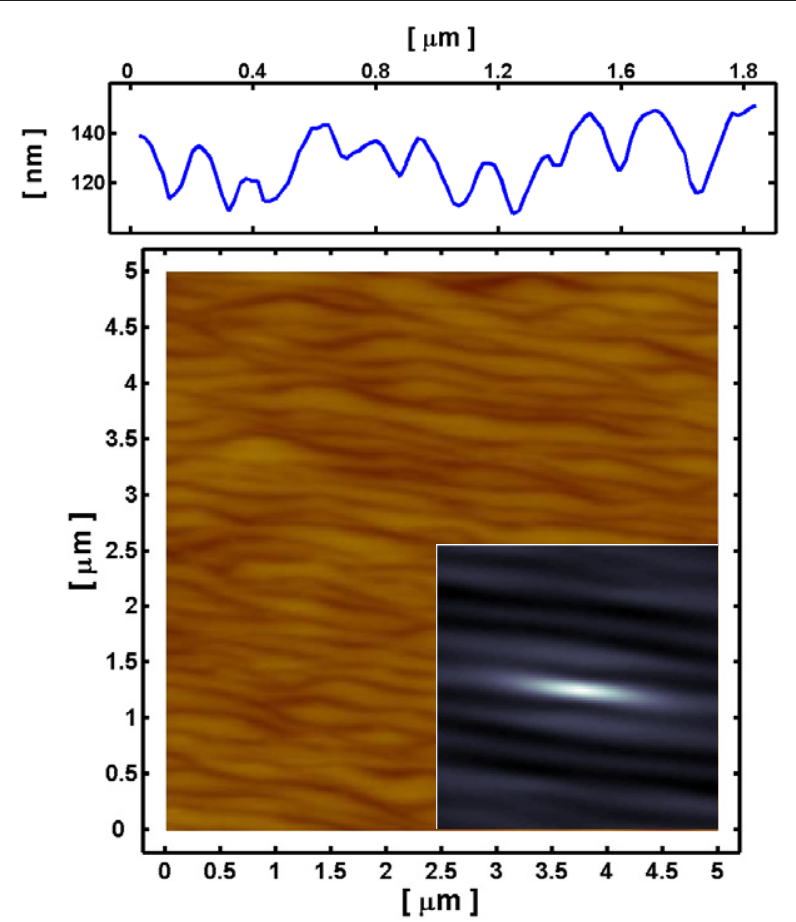

Figure 4. AFM two-dimensional view $\left(5 \times 5 \mu \mathrm{m}^{2}\right)$ of a nanostructured polymer and corresponding cross section. The inset shows the height-height correlation function $G(i, j)$ (area $\left.2 \times 2 \mu \mathrm{m}^{2}\right)$

smaller periodicities of about $10 \mathrm{~nm}$ are achieved on metal substrates [12-14]. In this case, since the RMS height of the features is correspondingly decreased, we expect an easy transfer of the pattern to the polymer surface by action of the capillary forces.

\section{Conclusion}

Summarizing, we have demonstrated that the combination of ion-sputtering and soft lithography can be an alternative and cheap way for nanostructuring soft matter. The selforganized nanoscale structures are created on a glass template by irradiating the surface with a defocused, low energy Ar ion beam. The use of capillary force lithography (CFL) allows us to transfer the pattern using the glass transition phenomenology of the polymeric layer. Specifically, we have demonstrated the transfer of a periodic pattern consisting in a ripple structure with a periodicity of $200 \mathrm{~nm}$. These nanostructures have a wide interest in view of their applications to both photonic patterned devices and organic nanoelectronics.

\section{References}

[1] Ross C B, Sun L and Crooks R M 1993 Langmuir 9632

[2] Xia Y and Whitesides G M 1998 Angew. Chem. Int. Edn Engl. 37550

[3] Chou S Y, Krauss P R and Renstrom P J 1995 Appl. Phys. Lett. 673114

[4] Chou S Y, Krauss P R and Renstrom P J 1996 Science 27285

[5] Kumar A and Whitesides G M 1993 Appl. Phys. Lett. 632002

[6] Mutzel M, Tandler S, Haubrich D, Meschede D, Peithmann K, Flaspohler M and Buse K 2002 Phys. Rev. Lett. 88083601

[7] Chai J, Li B and Kwok D Y 2005 Appl. Phys. Lett. 86034107

[8] Xia Y, Kim E, Zhao X-M, Rogers J A, Prentiss M and Whitesides G M 1996 Science 273347

[9] Zhao X-M, Xia Y and Whitesides G M 1996 Adv. Mater. 8837

[10] Kim E, Xia Y and Whitesides G M 1995 Nature 376581

[11] Kim E, Xia Y, Zhao X-M and Whitesides G M 1997 Adv. Mater. 9651

[12] Rusponi S, Costantini G, Buatier de Mongeot F, Boragno C and Valbusa U 1999 Appl. Phys. Lett. 753318

[13] Sekiba D, Bertero S, Buzio R, Buatier de Mongeot F, Boragno C and Valbusa U 2002 Appl. Phys. Lett. 812632

[14] Sekiba D, Moroni R, Gonella G, Buatier de Mongeot F, Boragno C, Mattera L and Valbusa U 2004 Appl. Phys. Lett. 84762

[15] Chason E, Mayer T M, Kellerman B K, Mcllroy D T and Howard A J 1994 Phys. Rev. Lett. 723040

[16] Carter G and Vishnyakov V 1996 Phys. Rev. B 5417647

[17] Bradley R M and Harper J M 1988 J. Vac. Sci. Technol. A 6 2390

[18] Flamm D, Frost F and Hirsch D 2001 Appl. Surf. Sci. 17995

[19] Umbach C, Headrick R L and Chang K C 2001 Phys. Rev. Lett. 87246104

[20] Toma A, Buatier de Mongeot F, Buzio R, Firpo G, Bhattacharyya S R, Boragno C and Valbusa U 2005 Nucl. Instrum. Methods B 230551

[21] Koponen I, Hautala M and Sievanen O P 1997 Phys. Rev. Lett. 782612

[22] Pisignano D, Persano L, Cingolani R, Gigli G, Barbudri F, Farinola G M and Naso F 2004 Appl. Phys. Lett. 841365

[23] Suh K Y and Lee H H 2002 Adv. Funct. Mater. 12405

[24] Chou S Y, Krauss P R, Zhang W, Guo L and Zhuang L 1997 J. Vac. Sci. Technol. B 152897

[25] Scheer H-C, Schulz H, Hoffmann T and Sotomayor Torres C M 1998 J. Vac. Sci. Technol. B 163917

[26] Mayers D 1999 Surface, Interface, and Colloids (New York: Wiley-VCH) pp 97-118

[27] Vogel H 1921 Phys. Z. 22645

Fulcher G S 1925 J. Am. Ceram. Soc. 8339

Tamman G and Hesse W 1926 Z. Anorg. Allg. Chem. 156245 